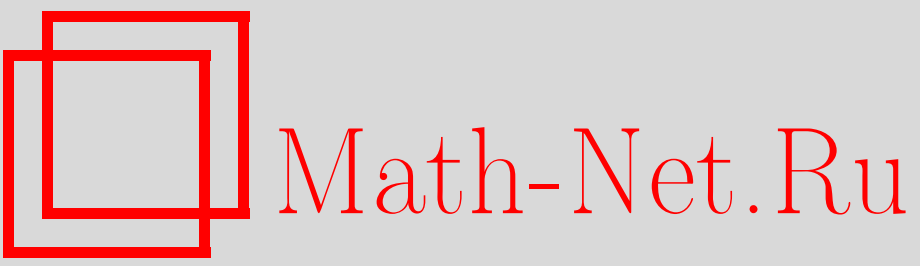

Д. А. Куликов, Динамика связанных осцилляторов Ван дер Поля, Итоги науки и техн. Сер. Соврем. мат. и ее прил. Темат. обз., 2019, том 168, 53-60

DOI: https://doi.org/10.36535/0233-6723-2019-168-53-60

Использование Общероссийского математического портала Math-Net.Ru подразумевает, что вы прочитали и согласны с пользовательским соглашением

http://www.mathnet.ru/rus/agreement

Параметры загрузки:

IP: 3.80 .181 .102

26 апреля 2023 г., 10:48:54 
ИТОГИ НАУКИ И ТЕХНИКИ.

Современная математика и ее приложения.

Тематические обзоры.

Том 168 (2019). C. $53-60$

DOI: $10.36535 / 0233-6723-2019-168-53-60$

УДК 517.929

\title{
ДИНАМИКА СВЯЗАННЫХ ОСЦИЛЛЯТОРОВ ВАН ДЕР ПОЛЯ
}

\author{
(c) 2019 г. Д. А. КУЛИКОВ
}

\begin{abstract}
АннотАция. В работе рассмотрена задача о синхронизации двух и трех слабосвязанных осцилляторов Ван дер Поля в случае, когда осцилляторы полностью идентичны и связь между ними слабая. Изучены вопросы существования и устойчивости двух типов периодических решений в предположении, что связь между осцилляторами диссипативна или активна. Анализ задачи базируется на использовании таких методов качественной теории дифференциальных уравнений, как метод интегральных многообразий и метод нормальных форм Пуанкаре-Дюлака. Задача сведена к изучению нормальных форм. При этом использована одна из версий алгоритма КрыловаБоголюбова, что позволило получать асимптотические формулы для периодических решений.
\end{abstract}

Ключевые слова: осциллятор, слабая связь, периодическое решение, устойчивость, нормальная форма, синхронизация.

\section{DYNAMICS OF COUPLED VAN DER POL OSCILLATORS}

\section{(c) 2019 D. A. KULIKOV}

\begin{abstract}
In this paper, we consider the problem on the synchronization of two and three weakly coupled Van der Pol oscillators in the case where the oscillators are identical and the connection between them is weak. The existence and stability of two types of periodic solutions are studied under the assumption that the connection between the oscillators is dissipative or active. The analysis of the problem is based on methods of the qualitative theory of differential equations, namely, the method of integral manifolds and the method of normal Poincaré-Dulac forms. The problem is reduced to the study of normal forms. We used a version of the Krylov-Bogolyubov algorithm, which allows one to obtain asymptotic formulas for periodic solutions.
\end{abstract}

Keywords and phrases: oscillator, weak connection, periodic solution, stability, normal form, synchronization.

AMS Subject Classification: 34C23, 34C25, 34D06

1. Введение. В теории нелинейных колебаний и радиофизике важную роль играет широко известное уравнение Ван дер Поля

$$
\ddot{x}-2 \varepsilon \dot{x}+x+a x^{2} \dot{x}=0,
$$

где $a>0$-постоянная и $\varepsilon>0$ рассматривается как малый параметр. Хорошо, известно, что уравнение (1) имеет устойчивый предельный цикл. Уравнение Ван дер Поля играет значительную роль в теории нелинейных колебаний, так как служит примером, пожалуй, простейшей автомодельной системы, в которой прослеживаются многие характерные свойства автоколебательных систем.

Работа выполнена при поддержке Российского фонда фундаментальных исследований (проект № 18-01-00672). 
Другой известной задачей следует считать задачу о синхронизации автоколебательных систем (см. $[4,5,10])$ при различных видах связи. В представленной работе будет изучена задача о синхронизации двух и трех осцилляторов Ван дер Поля при наличии слабой связи. Предполагается не только указать синхронные решения, но и исследовать вопрос об устойчивости таких решений.

Анализ задач предполагается провести при помощи метода нормальных форм. Такой подход позволяет изучить поставленные задачи в достаточно полном объеме, не упуская при этом вопрос об устойчивости. Данный подход к изучению явления синхронизации в системе слабосвязанных осцилляторов был использован в $[6-8,11]$. Более подробное изложение этого подхода к некоторым задачам теории синхронизации можно найти в диссертации [9].

2. Постановка задачи. В работе будут рассмотрены системы из двух и из трех дифференциальных уравнений второго порядка

$$
\left\{\begin{array}{l}
\ddot{x}_{1}-2 \varepsilon \dot{x}_{1}+x_{1}+a x_{1}^{2} \dot{x}_{1}=\varepsilon \gamma\left(\dot{x}_{2}-\dot{x}_{1}\right) \\
\ddot{x}_{2}-2 \varepsilon \dot{x}_{2}+x_{2}+a x_{2}^{2} \dot{x}_{2}=\varepsilon \gamma\left(\dot{x}_{1}-\dot{x}_{2}\right)
\end{array}\right.
$$

а также

$$
\left\{\begin{array}{l}
\ddot{x}_{1}-2 \varepsilon \dot{x}_{1}+x_{1}+a x_{1}^{2} \dot{x}_{1}=\varepsilon \gamma\left(\dot{x}_{3}-\dot{x}_{1}\right), \\
\ddot{x}_{2}-2 \varepsilon \dot{x}_{2}+x_{2}+a x_{2}^{2} \dot{x}_{2}=\varepsilon \gamma\left(\dot{x}_{1}-\dot{x}_{2}\right), \\
\ddot{x}_{3}-2 \varepsilon \dot{x}_{3}+x_{3}+a x_{3}^{2} \dot{x}_{3}=\varepsilon \gamma\left(\dot{x}_{2}-\dot{x}_{3}\right) .
\end{array}\right.
$$

Системы (2) и (3) представляют собой системы из двух и из трех слабосвязанных осцилляторов Ван дер Поля при наличии диссипативной (активной) связей. Связь считают диссипативной, если $\gamma>0$, и активной, если $\gamma<0$. Случай $\gamma=0$ не предполагается изучать, так как при таком варианте выбора $\gamma$ связь между осцилляторами отсутствует.

Отметим сразу, что системы $(2),(3)$ имеют периодические решения $x_{1}(t)=x_{2}(t)=y(t)$ в случае системы (2) (соответственно, $x_{1}(t)=x_{2}(t)=x_{3}(t)=y(t)$ в случае системы $(3)$, где $y(t)$ - цикл уравнения Ван дер Поля (1), удовлетворяющий уравнению

$$
\ddot{y}-2 \varepsilon y+y+a y^{2} \dot{y}=0, \quad a>0, \quad \varepsilon \in\left(0 ; \varepsilon_{0}\right) .
$$

Оба цикла в задачах теории колебаний принято называть синхронными (ииклами АндроноваХопфа). Если вопрос о существовании циклов Андронова-Хопфа в нашем случае очевиден и сводится к наличию циклов у уравнения Ван дер Поля (см. (4) и (1)), то вопрос об их устойчивости уже существенно содержательнее.

В работе также будет рассмотрен вопрос о существовании и устойчивости циклов, отличных от цикла Андронова-Хопфа, например, противофазного, в случае двух связанных осцилляторов. Напомним, что цикл системы дифференциальных уравнений (2) называют противофазным, если для него справедливо соотношение

$$
x_{2}(t, \varepsilon)=x_{1}(t+\pi, \varepsilon) .
$$

Добавим, что при $\varepsilon>0$ нулевые состояния равновесия систем (2) и (3) заведомо неустойчивы.

3. Два слабосвязанных осциллятора Ван дер Поля. Рассмотрим систему дифференциальных уравнений (2). Решения в достаточно малой окрестности нулевого состояния равновесия будем искать в следующем виде:

$$
\begin{aligned}
& x_{1}(t, s, \varepsilon)=\varepsilon^{1 / 2} u_{1}(t, s)+\varepsilon v_{1}(t, s)+\varepsilon^{3 / 2} w_{1}(t, s)+o\left(\varepsilon^{3 / 2}\right), \\
& x_{2}(t, s, \varepsilon)=\varepsilon^{1 / 2} u_{2}(t, s)+\varepsilon v_{2}(t, s)+\varepsilon^{3 / 2} w_{2}(t, s)+o\left(\varepsilon^{3 / 2}\right),
\end{aligned}
$$

где $s=\varepsilon t-$ медленное время,

$$
u_{1}(t, s)=z_{1}(s) \exp (i t)+\bar{z}_{1}(s) \exp (-i t), \quad u_{2}(t, s)=z_{2}(s) \exp (i t)+\bar{z}_{2}(s) \exp (-i t) .
$$

Функции $v_{1}, v_{2}, w_{1}, w_{2}$ имеют период $2 \pi$ по переменной $t$; кроме того, справедливы равенства

$$
M_{ \pm}\left(v_{j}\right)=\frac{1}{2 \pi} \int_{0}^{2 \pi} v_{j}(t, s) \exp ( \pm i t)=0, \quad M_{ \pm}\left(w_{j}\right)=\frac{1}{2 \pi} \int_{0}^{2 \pi} w_{j}(t, s) \exp ( \pm i t)=0, \quad j=1,2 .
$$


Отметим, что справедливо равенство

$$
\frac{d \psi}{d t}=\frac{\partial \psi}{\partial t}+\varepsilon \frac{\partial \psi}{\partial s}
$$

где $\psi(t, s)$ - некоторая гладкая функция аргументов $t$ и $s$. Далее точкой будем обозначать производную по $t$, а штрихом - по $s$.

Подставляя суммы (5) в систему (2) и приравнивая коэффициенты при $\varepsilon, \varepsilon^{3 / 2}$, получаем неоднородные системы дифференциальных уравнений. На втором шаге реализации алгоритма для $v_{1}, v_{2}$ получаем систему

$$
\ddot{v}_{1}+v_{1}=0, \quad \ddot{v}_{2}+v_{2}=0 .
$$

Эта система допускает периодическое решение $v_{1}=v_{2}=0$, для которого $M_{ \pm}\left(v_{j}\right)=0$.

На третьем шаге получаем систему из двух неоднородных дифференциальных уравнений

$$
\ddot{w}_{1}+w_{1}=F_{1}, \quad \ddot{w}_{2}+w_{2}=F_{2},
$$

где

$$
\begin{aligned}
& F_{1}=F_{1}\left(t, z_{1}, z_{2}\right)=-a u_{1}^{2} \dot{u}_{1}+2 \dot{u}_{1}+\gamma\left(\dot{u}_{2}-\dot{u}_{1}\right)-2 \dot{u}_{1}^{\prime}, \\
& F_{2}=F_{2}\left(t, z_{1}, z_{2}\right)=-a u_{2}^{2} \dot{u}_{2}+2 \dot{u}_{2}+\gamma\left(\dot{u}_{1}-\dot{u}_{2}\right)-2 \dot{u}_{2}^{\prime} .
\end{aligned}
$$

При анализе системы (6) следует интерпретировать вспомогательные переменные $z_{1}, z_{2}$ как параметры. Хорошо известно, что система (6) имеет $2 \pi$-периодические решения, если выполнены следующие условия:

$$
M_{ \pm}\left(F_{j}\right)=\frac{1}{2 \pi} \int_{0}^{2 \pi} F_{j} \exp ( \pm i t) d t=0, \quad j=1,2 .
$$

Добавим, что при этом решения $w_{1}, w_{2}$ следует выбрать таким образом, чтобы были справедливы равенства $M_{ \pm}\left(u_{j}\right)=0, j=1,2$. Из условий разрешимости в классе $2 \pi$-периодических по $t$ функций получаем, что комплекснозначные функции $z_{1}(s), z_{2}(s)$ должны удовлетворять системе дифференциальных уравнений (нормальных форм)

$$
\begin{aligned}
& z_{1}^{\prime}=z_{1}-\frac{a}{2} z_{1}\left|z_{1}\right|^{2}+\frac{\gamma}{2}\left(z_{2}-z_{1}\right) \\
& z_{2}^{\prime}=z_{2}-\frac{a}{2} z_{2}\left|z_{2}\right|^{2}+\frac{\gamma}{2}\left(z_{1}-z_{2}\right) .
\end{aligned}
$$

Для удобства положим $\gamma / 2=\mu$ и перепишем систему (7) в «тригонометрическом» виде, положив

$$
z_{1}=\sqrt{\frac{2}{a}} \rho_{1} \exp \left(i \varphi_{1}\right), \quad z_{2}=\sqrt{\frac{2}{a}} \rho_{2} \exp \left(i \varphi_{2}\right)
$$

где $\rho_{1}, \rho_{2} \geqslant 0\left(\rho_{1}=\rho_{2}=0\right.$ соответствует нулевому решению системы $\left.(7)\right)$. В результате получим систему действительных дифференциальных уравнений

$$
\left\{\begin{array}{l}
\rho_{1}^{\prime}=\rho_{1}-\rho_{1}^{3}+\mu\left(\rho_{2} \cos \left(\varphi_{2}-\varphi_{1}\right)-\rho_{1}\right), \\
\rho_{2}^{\prime}=\rho_{2}-\rho_{2}^{3}+\mu\left(\rho_{1} \cos \left(\varphi_{1}-\varphi_{2}\right)-\rho_{2}\right), \\
\varphi_{1}^{\prime}=\mu \frac{\rho_{2}}{\rho_{1}} \sin \left(\varphi_{2}-\varphi_{1}\right), \\
\varphi_{2}^{\prime}=\mu \frac{\rho_{1}}{\rho_{2}} \sin \left(\varphi_{1}-\varphi_{2}\right) .
\end{array}\right.
$$

Выполним в системе (8) подстановку $\Theta=\varphi_{2}-\varphi_{1}$ и для медленных переменных $\rho_{1}, \rho_{2}, \Theta$ получим замкнутую систему дифференциальных уравнений

$$
\left\{\begin{array}{l}
\rho_{1}^{\prime}=\rho_{1}-\rho_{1}^{3}+\mu\left[\rho_{2} \cos \theta-\rho_{1}\right], \\
\rho_{2}^{\prime}=\rho_{2}-\rho_{2}^{3}+\mu\left[\rho_{1} \cos \theta-\rho_{2}\right], \\
\Theta^{\prime}=-\mu \sin \theta\left[\frac{\rho_{1}}{\rho_{2}}+\frac{\rho_{2}}{\rho_{1}}\right] .
\end{array}\right.
$$


Очевидно, что каждому состоянию равновесия системы (9) соответствует периодическое решение системы (7). Поэтому центральным вопросом можно считать задачу о ненулевых состояниях равновесия системы дифференциальных уравнений (9).

Прежде чем перейти к анализу системы (9), отметим, что нулевое решение системы (7) неустойчиво при всех значениях параметра $\mu(\gamma)$.

Итак, найдем теперь ненулевые состояния равновесия системы дифференциальных уравнений (9). Получаем, что $\sin \Theta=0$. Это означает, что имеется два различных варианта выбора $\Theta$ : $\Theta_{1}=0, \Theta_{2}=\pi$.

В первом случае получаем состояние равновесия $S_{1}$, координаты которого равны

$$
\rho_{1}=\rho_{2}=1, \quad \Theta=0 .
$$

Второе состояние равновесия $S_{2}$ существует, если $1-2 \mu>0$; тогда

$$
\rho_{1}=\rho_{2}=\sqrt{1-2 \mu}, \quad \Theta=\pi .
$$

Следовательно, $S_{2}$ существует, если $\mu<1 / 2(\gamma<1)$.

Стандартная проверка показывает, что состояние равновесия $S_{1}: \rho_{1}=\rho_{2}=1, \Theta=0$ асимптотически устойчиво, если $\mu>0$, и неустойчиво, если $\mu<0$ (случай $\mu=0$ не рассматриваем, так как в нем отсутствует связь между осцилляторами).

Аналогичная проверка показала, что состояние равновесия $S_{2}=(\sqrt{1-2 \mu}, \sqrt{1-2 \mu}, \pi)$ устойчиво при $\mu<0$. При $\mu \in(0,1 / 2)$ оно существует, но неустойчиво.

Теорема 1. Существует такое $\varepsilon_{0}>0$, что при всех $\varepsilon \in\left(0, \varepsilon_{0}\right)$ система дифференииальных уравнений (2) имеет синхронное периодическое решение $\left(z_{1}=z_{2}\right)$, которое соответствует состоянию равновесия $S_{1}$ системы (9). Этот иикл орбитально асимптотически устойчив, если $\gamma>0(\mu>0)$.

Противофазный и,икл $\left(z_{2}=-z_{1}\right)$ существует, если $\gamma<1(\mu<1 / 2)$. Он устойчив при $\gamma<0 u$ неустойчив при $\gamma>0$.

Для решений, образующих синхронный чикл, справедлива асимптотическая формула

$$
\begin{aligned}
& x_{1}=2 \varepsilon^{1 / 2} \sqrt{\frac{2}{a}}\left[\cos \left(t+\varphi_{1}\right)\right]+O\left(\varepsilon^{3 / 2}\right), \\
& x_{2}=2 \varepsilon^{1 / 2} \sqrt{\frac{2}{a}}\left[\cos \left(t+\varphi_{1}\right)\right]+O\left(\varepsilon^{3 / 2}\right),
\end{aligned}
$$

где $\varphi_{1} \in \mathbb{R}$ и произвольно.

Для решений, образующих противофазный иикл, справедлива асимптотическое представление

$$
\begin{aligned}
& x_{1}=2 \varepsilon^{1 / 2} \sqrt{\frac{2}{a}} \sqrt{1-\gamma} \cos \left(t+\varphi_{2}\right)+O\left(\varepsilon^{3 / 2}\right), \\
& x_{2}=-2 \varepsilon^{1 / 2} \sqrt{\frac{2}{a}} \sqrt{1-\gamma} \cos \left(t+\varphi_{2}\right)+O\left(\varepsilon^{3 / 2}\right),
\end{aligned}
$$

где $\varphi_{2} \in \mathbb{R}$ произвольно.

4. Три связанных осциллятора. Рассмотрим систему дифференциальных уравнений (3), которую будем рассматривать как систему из трех слабосвязанных осцилляторов. Как и в предыдущем разделе, вопрос о поведении ее решений можно и целесообразно свести к аналогичным вопросам для вспомогательной системы дифференциальных уравнений для комплекснозначной функции, которую принято называть нормальной формой.

Будем искать решения системы дифференциальных уравнений в виде

$$
x_{j}(t, \varepsilon)=\varepsilon^{1 / 2} u_{j}(t, s)+\varepsilon v_{j}(t, s)+\varepsilon^{3 / 2}+o\left(\varepsilon^{3 / 2}\right),
$$

где $j=1,2,3, u_{j}(t, s)=z_{j} \exp (i t)+\bar{z}_{j} \exp (-i t), z_{j}=z_{j}(s), s=\varepsilon t, u_{j}(t, s)$ и $w_{j}(t, s)$ - достаточно гладкие функции, имеющие период $2 \pi$ по переменной $t$. Подставляя суммы $(10)$ в систему (3) и приравнивая коэффициенты при одинаковых степенях $\varepsilon\left(\varepsilon, \varepsilon^{3 / 2}\right)$, получим системы трех 
линейных неоднородных дифференциальных уравнений второго порядка для функций $v_{j}$ и $w_{j}$, $j=1,2,3$. Построения, аналогичные проведенным в предыдущем разделе, позволяют заключить, что $v_{1}=v_{2}=v_{3}=0$. Анализ системы неоднородных уравнений для $w_{1}, w_{2}, w_{3}$ показывает, что из условий их разрешимости в классе $2 \pi$-периодических по $t$ функций можно получить систему дифференциальных уравнений первого порядка для вспомогательных комплекснозначных функций $z_{1}(s), z_{2}(s), z_{3}(s)$. Эту систему и принято называть нормальной формой. Итак, нормальная форма в комплексной форме записи приобретает следующий вид:

$$
\left\{\begin{array}{l}
z_{1}^{\prime}=z_{1}-\frac{a}{2} z_{1}\left|z_{1}\right|^{2}+\frac{\gamma}{2}\left(z_{3}-z_{1}\right), \\
z_{2}^{\prime}=z_{2}-\frac{a}{2} z_{2}\left|z_{2}\right|^{2}+\frac{\gamma}{2}\left(z_{1}-z_{2}\right) \\
z_{3}^{\prime}=z_{3}-\frac{a}{2} z_{3}\left|z_{3}\right|^{2}+\frac{\gamma}{2}\left(z_{2}-z_{3}\right)
\end{array}\right.
$$

где $z_{k}^{\prime}=z_{k}^{\prime}(s)$ - производная по переменной $s$. Положим $\mu=\gamma / 2$ и

$$
z_{j}(s)=\sqrt{\frac{2}{a}} \rho_{j}(s) \exp \left(i \varphi_{j}(s)\right)
$$

т.е. перейдем к нормированному варианту записи комплекснозначных функций. В результате подстановки равенств (12) в систему (11) и разделения действительных и мнимых частей получаем новый вариант записи системы (11):

$$
\left\{\begin{array}{l}
\rho_{1}^{\prime}=\rho_{1}-\rho_{1}^{3}+\mu\left[\rho_{3} \cos \left(\varphi_{3}-\varphi_{1}\right)-\rho_{1}\right] \\
\rho_{2}^{\prime}=\rho_{2}-\rho_{2}^{3}+\mu\left[\rho_{1} \cos \left(\varphi_{1}-\varphi_{2}\right)-\rho_{2}\right], \\
\rho_{3}^{\prime}=\rho_{3}-\rho_{3}^{3}+\mu\left[\rho_{2} \cos \left(\varphi_{2}-\varphi_{3}\right)-\rho_{3}\right], \\
\varphi_{1}^{\prime}=\mu \frac{\rho_{3}}{\rho_{1}} \sin \left(\varphi_{3}-\varphi_{1}\right), \\
\varphi_{2}^{\prime}=\mu \frac{\rho_{1}}{\rho_{2}} \sin \left(\varphi_{1}-\varphi_{2}\right), \\
\varphi_{3}^{\prime}=\mu \frac{\rho_{2}}{\rho_{3}} \sin \left(\varphi_{2}-\varphi_{3}\right),
\end{array}\right.
$$

т.е. систему, состоящую из трех уравнений для амплитудных переменных и трех уравнений для аргументов $\varphi_{1}, \varphi_{2}, \varphi_{3}$.

Положим теперь

$$
\varphi_{3}-\varphi_{1}=\Theta_{1}, \quad \varphi_{1}-\varphi_{2}=\Theta_{2}
$$

Тогда $\varphi_{2}-\varphi_{3}=-\Theta_{1}-\Theta_{2}$. Для $\rho_{1}, \rho_{2}, \rho_{3}, \Theta_{1}, \Theta_{2}$ можно получить замкнутую систему дифференциальных уравнений

$$
\left\{\begin{array}{l}
\rho_{1}^{\prime}=\rho_{1}-\rho_{1}^{3}+\mu\left[\rho_{3} \cos \Theta_{1}-\rho_{1}\right] \\
\rho_{2}^{\prime}=\rho_{2}-\rho_{2}^{3}+\mu\left[\rho_{1} \cos \Theta_{2}-\rho_{2}\right] \\
\rho_{3}^{\prime}=\rho_{3}-\rho_{3}^{3}+\mu\left[\rho_{2} \cos \left(\Theta_{1}+\Theta_{2}\right)-\rho_{3}\right] \\
\Theta_{1}^{\prime}=-\mu\left[\frac{\rho_{2}}{\rho_{3}} \sin \left(\Theta_{1}+\Theta_{2}\right)+\frac{\rho_{3}}{\rho_{1}} \sin \Theta_{1}\right] \\
\Theta_{2}^{\prime}=\mu\left[\frac{\rho_{3}}{\rho_{1}} \sin \Theta_{1}-\frac{\rho_{1}}{\rho_{2}} \sin \Theta_{2}\right]
\end{array}\right.
$$

Замкнутую систему (14) следует дополнить любым уравнением из последних трех уравнений системы (13). Очевидно, что любому состоянию равновесия вспомогательной системы дифференциальных уравнений (14) соответствует периодическое решение нормальной формы (11). Найдем состояния равновесия системы (14), представляющие наибольший интерес с прикладной точки зрения. Так, система (14) имеет состояние равновесия

$$
S_{3}: \quad \rho_{1}=\rho_{2}=\rho_{3}=1, \quad \Theta_{1}=\Theta_{2}=0,
$$


которое соответствует состоянию равновесия $S_{1}$ из предыдущего раздела, а также состояние равновесия

$$
S_{4}: \quad \rho_{1}=\rho_{2}=\rho_{3}=\left(1-\frac{3}{2} \mu\right)^{1 / 2}, \quad \Theta_{1}=\Theta_{2}=\frac{2 \pi}{3},
$$

которое существует, если $1-3 \mu / 2>0$, т.е. при $\mu<2 / 3(\gamma<4 / 3)$. Состояние равновесия $S_{3}$ существует при всех $\mu(\gamma)$.

Рассмотрим вопрос об устойчивости состояний равновесия $S_{3}, S_{4}$. Для этого нужно изучить расположение собственных значений матриц $B_{3}$ и $B_{4}$, получающихся линеаризацией системы дифференциальных уравнений (14) на состояниях равновесия $S_{3}$ и $S_{4}$ соответственно.

Стандартные вычисления позволяют вычислить элементы матриц $B_{3}, B_{4}$. Матрица $B_{3}$ имеет блочный вид

$$
B_{3}=\operatorname{diag}\left(B_{31}, B_{32}\right), \quad B_{31}=\left(\begin{array}{ccc}
-2-\mu & 0 & \mu \\
\mu & -2-\mu & 0 \\
0 & \mu & -2-\mu
\end{array}\right), \quad B_{32}=\left(\begin{array}{cc}
-2 \mu & -\mu \\
\mu & -\mu
\end{array}\right) .
$$

Элементарные вычисления показывают, что все собственные значения матрицы $B_{3}$ лежат в левой полуплоскости комплексной плоскости $(\operatorname{Re} \lambda<0)$, если $\mu>0$ и, напротив, существует, по крайней мере одно собственное число, лежащее в правой полуплоскости комплексной плоскости, если $\mu<0(\mu \neq 0)$ по условию.

Аналогичные вычисления показывают, что матрица

$$
B_{4}=\left(\begin{array}{ccccc}
-2+\frac{7}{2} \mu & 0 & -\frac{\mu}{2} & -\sqrt{3} \eta \frac{\mu}{2} & 0 \\
-\frac{\mu}{2} & -2+\frac{7}{2} \mu & 0 & 0 & -\sqrt{3} \eta \frac{\mu}{2} \\
0 & -\frac{\mu}{2} & -2+\frac{7}{2} \mu & \sqrt{3} \eta \frac{\mu}{2} & \sqrt{3} \eta \frac{\mu}{2} \\
\frac{\sqrt{3}}{\eta} \frac{\mu}{2} & \frac{\sqrt{3}}{\eta} \frac{\mu}{2} & -\mu \frac{\sqrt{3}}{\eta} & \mu & \frac{\mu}{2} \\
-\mu \frac{\sqrt{3}}{\eta} & \frac{\mu}{2} \frac{\sqrt{3}}{\eta} & \frac{\mu}{2} \frac{\sqrt{3}}{\eta} & -\frac{\mu}{2} & \frac{\mu}{2}
\end{array}\right),
$$

где $\eta=\sqrt{1-3 \mu / 2}$. Вычисления показывают, что при $\mu<0$ все собственные значения лежат в левой полуплоскости комплексной плоскости $(\operatorname{Re} \lambda<0)$, но при $\mu>0$ у данной матрицы имеются собственные значения с $\operatorname{Re} \lambda>0$. Проверка этого утверждения опиралась, в частности, на использования критерия Рауса-Гурвица.

Приведенные рассуждения вместе с результатами работ $[2,3]$ приводят к следующему утверждению.

Теорема 2. Существует такое $\varepsilon_{0}>0$, что при всех $\varepsilon \in\left(0, \varepsilon_{0}\right)$ система дифференциальных уравнений (11) имеет периодические решения, соответствующие состояниям равновесия $S_{3}$, $S_{4}$ системы дифференциальных уравнений (14).

Состоянию равновесия $S_{3}$ соответствует иикл $C_{3}$ :

$$
\begin{aligned}
& x_{1}(t, \varepsilon)=2 \sqrt{\frac{2}{a}} \varepsilon^{1 / 2} \cos \left(t+\psi_{1}\right)+O\left(\varepsilon^{3 / 2}\right), \\
& x_{2}(t, \varepsilon)=2 \sqrt{\frac{2}{a}} \varepsilon^{1 / 2} \cos \left(t+\psi_{1}\right)+O\left(\varepsilon^{3 / 2}\right), \\
& x_{3}(t, \varepsilon)=2 \sqrt{\frac{2}{a}} \varepsilon^{1 / 2} \cos \left(t+\psi_{1}\right)+O\left(\varepsilon^{3 / 2}\right),
\end{aligned}
$$

где $\psi_{1} \in \mathbb{R}$. Данное периодическое решение существует, если а > 0, при любом выборе $\gamma$. При $\gamma>0$ эти решения устойчивы (орбитально асимптотически устойчивы; см. [1]). 
Состоянию равновесия $S_{4}$ соответствует иикл $C_{4}$ :

$$
\begin{aligned}
& x_{1}(t, \varepsilon)=2 \sqrt{\frac{2}{a}} \sqrt{1-\frac{3 \gamma}{4}} \varepsilon^{1 / 2} \cos \left(\left(1+\varepsilon \frac{\sqrt{3} \gamma}{4}\right) t+\psi_{2}\right)+O\left(\varepsilon^{3 / 2}\right), \\
& x_{2}(t, \varepsilon)=2 \sqrt{\frac{2}{a}} \sqrt{1-\frac{3 \gamma}{4}} \varepsilon^{1 / 2} \cos \left(\left(1+\varepsilon \frac{\sqrt{3} \gamma}{4}\right) t+\psi_{2}-\frac{2 \pi}{3}\right)+O\left(\varepsilon^{3 / 2}\right), \\
& x_{3}(t, \varepsilon)=2 \sqrt{\frac{2}{a}} \sqrt{1-\frac{3 \gamma}{4}} \varepsilon^{1 / 2} \cos \left(\left(1+\varepsilon \frac{\sqrt{3} \gamma}{4}\right) t+\psi_{2}+\frac{2 \pi}{3}\right)+O\left(\varepsilon^{3 / 2}\right),
\end{aligned}
$$

где $\psi_{2} \in \mathbb{R}$ произвольно. Цикл $C_{4}$ существует при выполнении неравенства $\gamma<4 / 3$, а устойчив (орбитально асимптотически устойчив) при $\gamma<0$.

5. Заключение. Рассмотрена задача о синхронизации автоколебаний в системе из двух и трех осцилляторов Ван дер Поля в случае активной или диссипативной связи (в терминологии работ [4, $5,10])$. Всегда существует синхронный цикл (цикл Андронова-Хопфа), и он устойчив при $\gamma>0$ (случай диссипативной связи).

В системе из двух связанных осцилляторов имеется противофазный цикл, для которого справедливо соотношение $x_{2}(t, \varepsilon)=x_{1}(t+\pi, \varepsilon)=-x_{1}(t, \varepsilon)$. В случае трех осцилляторов имеет место «подправка». Существует цикл $C_{4}$, который, вероятно, можно интерпретировать как аналог противофазного. Для него характерно следующее: колебания компонент сдвинуты по фазе на $2 \pi / 3$, а не на $\pi$, как в случае двух осцилляторов.

В данной работе рассматривался случай диссипативной (активной) связи. Иная задача возникает, когда имеет место инерционная связь (см. $[4,5,10])$. Например, в случае двух осцилляторов следует рассмотреть систему

$$
\left\{\begin{array}{l}
\ddot{x}_{1}-2 \varepsilon \dot{x}_{1}+x_{1}+a x_{1}^{2} \dot{x}_{1}=\gamma\left(x_{2}-x_{1}\right), \\
\ddot{x}_{2}-2 \varepsilon \dot{x}_{2}+x_{2}+a x_{2}^{2} \dot{x}_{2}=\gamma\left(x_{1}-x_{2}\right) .
\end{array}\right.
$$

В системе (16) по сравнению с системой (2) предложено изменение вида связи, правых частей уравнений. Следует отметить, что в $[7,8]$ была изучена система $(16)$, анализ которой показал, что динамика решений системы существенно богаче, чем динамика решений системы (2). Этого следует ожидать, по-видимому, и в случае трех осцилляторов, т.е. при аналогичном изменении правых частей системы дифференциальных уравнений (3).

\section{СПИСОК ЛИТЕРАТУРЫ}

1. Демидович Б. П. Лекции по математической теории устойчивости. - М.: Наука, 1967.

2. Колесов А. Ю., Куликов А. Н., Розов Н. Х. Инвариантные торы одного класса точечных отображений: принцип кольца// Диффер. уравн. - 2003. - 39, № 5. - С. 584-601.

3. Колесов А. Ю., Куликов А. Н., Розов Н. Х. Инвариантные торы одного класса отображений: сохранение тора при возмущениях// Диффер. уравн. - 2003. - 39, № 6. - С. 738-752.

4. Кузнецов А. П., Паксютов В. И. О динамике двух слабосвязанных осцилляторов Ван дер ПоляДуффинга с диссипативной связью// Изв. вузов. Прикл. нелин. динам. -2003 . - 11, № 6. - С. 48-63.

5. Кузнещов А. П., Тюрюкина Л. В., Сатаев И. Р., Чернышов Н. Ю. Синхронизация и многочастотные колебания в низкоразмерных ансамблях осцилляторов// Изв. вузов. Прикл. нелин. динам. - 2014. 22 , № 1. - C. $27-54$.

6. Куликов Д. А. Автомодельные периодические решения и бифуркации от них в задаче о взаимодействии двух слабосвязанных осцилляторов// Изв. вузов. Прикл. нелин. динам. $-2006 .-14$, № 5 . C. $120-132$.

7. Куликов Д. А. Циклы билокальной модели волнового уравнения: полный анализ// Соврем. пробл. мат. информ. - 2001. - 4. - С. 93-96.

8. Куликов Д. А. Исследование динамики билокальной модели нелинейных волновых уравнений// Соврем. пробл. мат. информ. - 2002. - 5. - С. 46-52.

9. Куликов Д. А. Аттракторы уравнения Гинзбурга-Ландау и его конечномерного аналога/ Дисс. на соиск. уч. степ. канд. физ.-мат. наук, 2006. 
10. Пиковский А., Розенблюм М., Курти Ю. Синхронизация. Фундаментальное явление. - М.: Техноcфepa, 2003.

11. Kulikov D. A. Self-similar cycles and their local bifurcations in the problem of two weakly coupled oscillators// J. Appl. Math. Mech. - 2010. — 74, № 4. - P. 389-400.

Куликов Дмитрий Анатольевич

Ярославский государственный университет им. П. Г. Демидова

E-mail: kulikov_d_a@mail.ru 